\title{
FLORA FÚNGICA CONTAMINANTE NATURAL DE ARÁNDANOS (Vaccinium corymbosum L. O'Neal) FRESCOS Y TRATADOS TÉRMICAMENTE
}

\author{
Rivas, M. Z. ${ }^{1,2}$; PIAgentini, A. M. ${ }^{2} \&$ Frisón, L. N. ${ }^{1}$
}

\begin{abstract}
RESUMEN
Se realizó el aislamiento e identificación de los mohos presentes en arándanos frescosy tratados térmicamente $\left(10\right.$ minutos a $80^{\circ} \mathrm{C}$ ), y se determinó su Abundancia (Ab) y Frecuencia (Fr).Se aislaron e identificaron ocho especies de mohos en arándanos frescos y ocho especies de mohos en arándanos tratados térmicamente (mohos termorresistentes). Las especies de mohos aislados de arándanos frescos más frecuentes y abundantes fueron Aspergillus niger (Fr: 70\% y Ab: 31\%) y Penicillium decumbens (Fr: 21\% y Ab: 11\%). Las especies de mohos aislados de arándanos tratados térmicamente más frecuentes y abundantes fueron Arthrinium phaeospermun (Fr: 30\% y Ab: 36\%) y Eurotium repens (Fr: 13\% y Ab: 21\%). Los resultados obtenidos sirven como base para el diseño de tratamientos postcosecha que permitan reducir los mohos más frecuentes y abundantes presentes tanto en arándanos frescos como en aquellos destinados a procesamientos que incluyan una etapa de tratamiento térmico.

Palabras clave: arándanos, hongos, contaminación.
\end{abstract}

\begin{abstract}
Naturally contaminating fungal flora of fresh and heat-treated blueberries (Vaccinium corymbosum L. O'Neal).

Isolation and identification of molds present in fresh and heat-treated blueberries $(10 \mathrm{mi}-$ nutes at $80^{\circ} \mathrm{C}$ ) were performed, and their Abundance (Ab) and Frequency (Fr) were determined. Eight species of molds in fresh blueberries and eight species of heat-resistant molds in heat-treated blueberries were isolated and identified. The most frequent and abundant molds isolated in fresh blueberry were Aspergillus niger (Fr: 70\% and Ab: 31\%) and Penicillium decumbens (Fr: 21\% and Ab: 11\%). The most frequent and abundant heat-resistant molds were $\mathrm{Ar}$ -
\end{abstract}

1.- Cátedra de Microbiología. Facultad de Ingeniería Química, Universidad Nacional del Litoral. (3000). Santa Fe. Argentina. Tel.03424571164 int.2541. Email: Ifrison@fiq.unl.edu.ar

2.- Instituto de Tecnología de Alimentos - Facultad de Ingeniería Química - Universidad Nacional del Litoral. (3000). Santa Fe. Argentina.

Manuscrito recibido el 16 de octubre de 2019 y aceptado para su publicación el 27 de noviembre de 2019.

Rivas, M. Z.; Piagentini A. M.; Frisón, L.N. Flora fúngica contaminante natural de arándanos (Vaccinium corymbosum L. O’Neal) frescos y tratados térmicamente. FAVE - Ciencias Agrarias 18 (1): 63-72. CC BY-NC-SA 4.0 (c) (i) (2) 


\section{Z. Rivas et al.}

thrinium phaeospermun (Fr: 30\% and Ab: 36\%) and Eurotium repens (Fr: 13\% and Ab: 21\%). These results serve as the basis for designing post-harvest treatments for reducing the most frequent and abundant molds present in both blueberries, fresh and those intended for processing.

Key words: blueberries, molds, contamination.

\section{INTRODUCCIÓN}

La contaminación superficial de frutas y hortalizas varía en número y tipo, dependiendo del producto y del manejo previo y posterior a la cosecha que dicho producto haya recibido. Los mecanismos de defensa de la fruta son altamente efectivos contra casi todos los hongos, por lo que relativamente pocos géneros y especies son aptos para invadir y causar severas pérdidas (18).

Los problemas de contaminación causados por mohos resistentes al calor, han existido desde que el hombre comenzó a preservar las frutas por un proceso térmico (19). Muchos de ellos producen ascosporas que pueden permanecer en estado latente por meses y aún por años en el suelo, en los recipientes y equipos utilizados para su cosecha, transporte y procesamiento, y las mismas pueden sobrevivir a los tratamientos térmicos, ya que las condiciones en que se realizan los mismos para productos elaborados a partir de frutas, no tienen la relación tiempo-Temperatura necesaria para su eliminación, debido a que dichas condiciones producirían grandes pérdidas de calidad sensorial y nutricional. Es por eso que si frutas con mohos resistentes al calor superan las etapas previas de selección y desinfección, posteriormente pueden germinar dentro de los productos envasados causando pérdidas económicas (28).

Las frutas se vuelven cada vez más susceptibles a la invasión de microorganismos durante la maduración, ya que el pH del tejido aumenta y las barreras de defensa se debilitan, entre otros factores. Por lo que es necesario reducir la contaminación de microorganismos patógenos que puedan afectar la salud del consumidor y la de los microorganismos alterantes que puedan afectar la calidad del producto durante el almacenamiento postcosecha $(28,8)$.

Hay un notable cambio en las pautas de consumo hacia productos naturales con componentes benéficos para la salud. Dietas ricas en antioxidantes están asociadas con un menor riesgo de padecer patologías cardiovasculares, neurodegenerativas, cáncer e incluso el envejecimiento, todas ellas vinculadas al estrés oxidativo (14). El consumo frutas y hortalizas aporta vitaminas, minerales, fibras y compuestos biológicamente activos, responsables de su potencial saludable, que también influyen en el color y sabor de estos productos $(4,9)$.

Los arándanos pertenecen al género Vaccinium de la familia de las Ericáceas. De las más de 30 especies del género Vaccinium sólo un pequeño grupo tiene importancia comercial. En nuestro país las especies cultivadas son $V$. corymbosum (highbush o arándano alto) y $V$. ashei (rabbiteye u ojo de conejo). Conforman un grupo de frutas denominadas comercialmente como "frutos del bosque”, entre las que se encuentran la frutilla, frambuesa y mora, entre otras (23, 11). El valor económico del arándano está en constante crecimiento, dado el incremento 
en la demanda por parte de los consumidores incluyéndolo en su dieta diaria. Esto se debe a que este fruto ha sido reconocido como un alimento saludable, debido a su elevado valor nutricional y terapéutico $(1,21)$. Desde el punto de vista nutricional, los arándanos tienen compuestos con gran capacidad antioxidante como antocianinas y compuestos fenólicos, responsables también del color rojo-azulado característico de su piel (12). Los polifenoles, entre ellos los flavonoides y en particular las antocianinas, poseen gran capacidad antioxidante; existiendo correlación positiva entre la actividad antioxidante, los polifenoles totales y/o el contenido de antocianinas $(5,3,13)$.

El arándano es un cultivo de creciente interés en Argentina. Se trata de un cultivo intensivo en inversión y en mano de obra. Es de gran importancia la producción y exportación de fruta fresca a nivel nacional (23). Las principales provincias productoras son: Entre Ríos, Tucumán y Buenos Aires, y en menor proporción San Luis, Río Negro, Mendoza, Salta, Catamarca y San Juan. En la provincia de Entre Ríos, las plantaciones se centran principalmente en la ciudad de Concordia y en la Región de Salto Grande, cubriendo, aproximadamente, el 50\% de la producción nacional, seguido por Federación y Gualeguaychú (24). Su cultivo es estacional (desde septiembre a mayo). Aproximadamente el 90\% de la producción nacional de arándanos se exporta en fresco al hemisferio norte, principalmente a Estados Unidos y a algunos de países de Europa como el Reino Unido, aprovechando los beneficios de la contra estación $(22,5)$. El resto de divide entre el mercado interno como fruta fresca, y como fruta congelada para la elaboración de confituras, helados, jugos concentrados, lácteos, entre otros. $(24,16)$.
La cosecha en condiciones de alta humedad ambiental (rocío, lluvia) y temperatura, aumenta la posibilidad de infección de los frutos por microorganismos durante la postcosecha. En estas condiciones, el deterioro de los frutos puede observarse en menos de 12 horas (3).

Los arándanos constituyen un grupo de especies nativas principalmente del hemisferio norte; siendo su habitad natural las regiones frías de Norteamérica, Europa Central y Asia, aunque también hay especies adaptadas a zonas tropicales de América Latina y África. (10). En las últimas décadas del siglo pasado, fueron introducidos al país, por lo cual no cuentan con enfermedades o plagas que sean propias de este cultivo; sin embargo las enfermedades fúngicas junto a la mosca de la fruta son las principales causales de pérdidas en los cultivos (16).

El objetivo de este trabajo fue estudiar la diversidad fúngica de arándanos frescos (Vaccinium corymbosum L. O'Neal) y tratados térmicamente (mohos termorresistentes), y determinar la abundancia y la frecuencia de cada uno de los mohos aislados e identificados.

\section{MATERIALES Y MÉTODOS}

\section{Muestras}

Se estudiaron 10 muestras de arándanos azules frescos (Familia Ericaceae, género Vaccinium L., especie corymbosum, variedad $\left.O^{\prime} N e a l\right)$, sin daño fisiológico aparente, con un diámetro promedio de 1,32 $\pm 0,14$ $\mathrm{cm}$ y un peso individual promedio de 1,35 $\pm 0,39$ g, y 10 muestras de arándanos azules tratados térmicamente $\left(10 \mathrm{~min}\right.$ a $\left.80^{\circ} \mathrm{C}\right) . \mathrm{El}$ estudio se realizó durante el período 20142015, con arándanos provenientes de una plantación comercial de la zona de Concor- 
dia, provincia de Entre Ríos, cosechados en forma manual y transportados inmediatamente hasta el laboratorio a $1,5^{\circ} \mathrm{C}$, para su análisis. Cada una de las muestras fue de 300 - $350 \mathrm{~g}$.

\section{Aislamiento de la flora fúngica presente en arándanos frescos sin tratamiento térmico (STT)}

En condiciones de esterilidad, se tomaron $100 \mathrm{~g}$ de cada muestra de arándanos frescos. Dado que la contaminación fúngica puede no ser homogénea en la muestra, se trituraron y homogeneizaron en forma estandarizada durante $60 \mathrm{~s}$ en Stomacher (Lab - Blender 400, Inglaterra) hasta la formación de una masa homogénea (19).

La fruta homogeneizada se mezcló, en una proporción 1:1, con medio MEA (Extracto de Malta Agar) de doble concentración, al cual se le agregó cloranfenicol 100 $\mathrm{mg} \mathrm{L}^{-1}$ para inhibir el desarrollo bacteriano. Luego se distribuyó la mezcla en Placas de Petri estériles. Las mismas se dejaron solidificar bajo la corriente de aire estéril en el flujo laminar (Casiba HL2B, Ind. Argentina bajo licencia de FARR Co., Los Ángeles, California U.S.A.). Las placas de Petri se colocaron en bolsas de polietileno para prevenir la evaporación y se incubaron durante 7 días a $25^{\circ} \mathrm{C}(28,19,7)$.

Para poder obtener cultivos puros, libre de contaminación y listos para la identificación de especies, cada una de las colonias, fueron aisladas por inoculación con ansa aguja de pequeños trozos de micelio o esporas a placas con medio de cultivo MEA. Luego de ser incubadas hasta su esporulación a $25^{\circ} \mathrm{C}$, se constató la pureza de las colonias por la uniformidad de apariencia de las mismas.

\section{Aislamiento de la flora fúngica resisten- te al calor en arándanos con tratamiento térmico (CTT) \\ Las muestras fueron tratadas de igual} manera que en el caso de los arándanos STT, hasta la formación de un una masa homogénea. Se tomó una alícuota de 100 g del puré de arándano, se colocó en un frasco estéril con tapa de $500 \mathrm{ml}$ y se sometió a un tratamiento térmico durante 10 minutos a $80^{\circ} \mathrm{C}$ en un baño de agua con agitación de la muestra. Luego se enfrió rápidamente en baño de agua fría (25).

En estudios realizados con anterioridad, se comprobó que a la temperatura seleccionada para la realización de este tratamiento térmico, permite muy buena activación de las ascosporas de los mohos perfectos (resistentes a los tratamientos térmicos) y se logra eliminar los mohos no termorresistentes (7). Para la siembra se procedió de igual manera que en el caso de los arándanos frescos STT.

El medio MEA con antibiótico (cloranfenicol) fue elegido por estar recomendado dentro del protocolo especial para el aislamiento de mohos resistentes al calor (19).

Se procedió a la incubación y al aislamiento de igual manera que en el caso de los arándanos frescos STT.

\section{Identificación de la flora fúngica}

La identificación de los mohos aislados presentes en arándanos CTT y STT se realizó mediante el cultivo en medios específicos y la observación de las características macroscópicas y microscópicas en distintas condiciones de cultivo y mediante el uso de las claves taxonómicas de Pitt y Hocking (2009). Para determinar las características macroscópicas de los aislados seleccionados, se dejaron crecer en medios de culti- 
vos CYA (Czapek yeast extract agar), MEA (agar extracto de malta) y G25N (25\% Glycerol nitrate agar) y se determinó el diámetro de las colonias a tiempos determinados, la textura y el color de las colonias entre otras características. Las características microscópicas, como morfología de los conidióforos; forma y tamaño de los conidios entre otras, fueron determinadas mediante observaciones en microscopio óptico.

Los cultivos a identificar se desarrollaron durante 7 días en los siguientes tres medios de cultivo: CYA a 5, 25 y $37^{\circ} \mathrm{C}$; MEA a $25^{\circ} \mathrm{C}$; G25N a $25^{\circ} \mathrm{C}$.

La conservación de los mohos aislados e identificados STT y CTT se realizó en crioviales conteniendo Agar-Agua (0,2 \% mv $\left.{ }^{-1}\right)$ y a temperatura de $7^{\circ} \mathrm{C}$.

\section{Determinación de la Abundancia (Ab) y la Frecuencia (Fr) de mohos aislados}

Con los mohos aislados e identificados de arándanos STT y CTT, se procedió a la determinación del porcentaje de Abundancia (Ab) y el porcentaje de Frecuencia (Fr), que se definen como:

Abundancia: número de colonias que se obtuvieron de cada especie de moho determinada sobre el total de colonias aisladas, por 100. Se expresa como:

Abundancia $(\mathrm{Ab})=\left(\mathrm{n}^{\circ}\right.$ ufc de cada especie de moho determinada / $\mathrm{n}^{\circ}$ ufc Totales) x 100

Frecuencia: número de veces que aparece una colonia de una especie de moho determinada sobre el total de muestras estudiadas, por 100. Se expresa como:

Frecuencia $(\mathrm{Fr})=\left(\mathrm{n}^{\circ}\right.$ veces que aparece una especie de moho determinada $/ \mathrm{n}^{\circ}$ de muestras estudiadas) x 100

\section{RESULTADOS}

En las muestras de arándanos frescos (STT) y con tratamiento térmico (CTT) se aislaron las siguientes especies de mohos: Arthrinium phaeospermun, Aspergillus niger, Bysochlamys nivea, Eurotium repens, Fusarium sporotrichioides, Monascus ruber, Neosartorya fischeri, Nigrospora oryzae, Penicillium pinophilum, Penicillium decumbens, Penicillium purpurogenum, Rhizopus microsporus, Rhizopus oryzae, Talaromyces bacillusporus, Talaromyces flavus.

\section{Especies de mohos aislados en arándanos STT}

En la Figura 1 se pueden observar los valores de Ab y Fr obtenidos de las especies de mohos aisladas de arándanos STT.

Con los datos obtenidos tanto de Fr como de $\mathrm{Ab}$ podemos decir que la especie $A$. niger presenta el mayor porcentaje en ambos casos, siendo el más frecuente (70\%) y abundante (31\%) de todas las especies aisladas en las muestras de arándanos STT analizadas. Sigue en segundo lugar $P$. decumbens, con un porcentaje menor en relación a la Fr (21\%) y la Ab (11\%). En tercer lugar se ubica a $P$. purpurogenum con un valor de $14 \%$ de Fr y una Ab por debajo de $10 \%$, al igual que el resto de las especies aisladas. Si bien las levaduras se aislaron de forma frecuente (14\%) y fueron muy abundantes (32\%) las mismas no fueron objeto de este estudio.

Los resultados obtenidos (Figura 1), mostraron que $A$. niger fue el que presentó el mayor porcentaje de Ab y Fr en los arándanos frescos estudiados, concordando con lo reportado por Plancich (2013), quien 


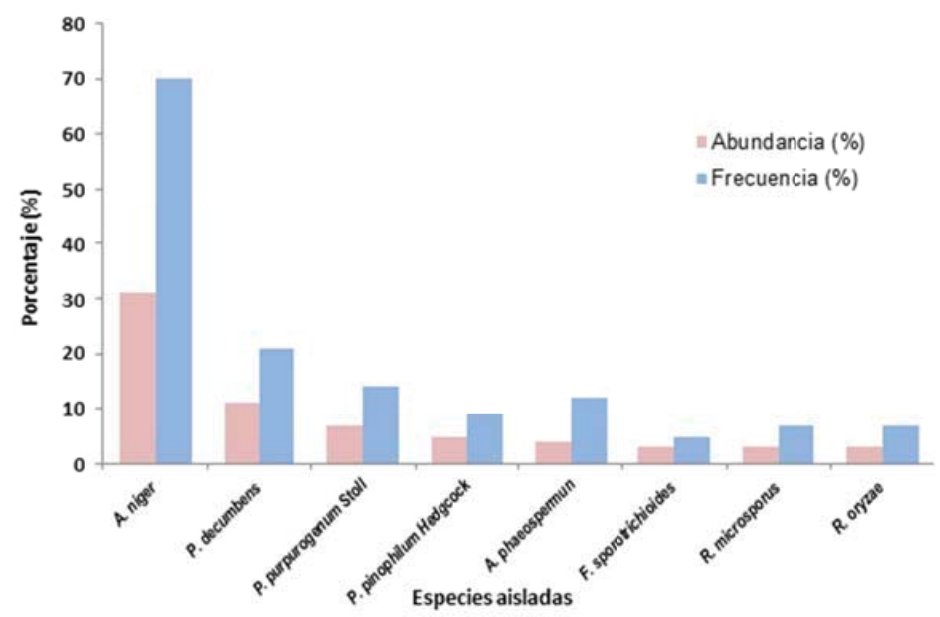

Figura 1. Abundancia y Frecuencia (\%) de mohos aislados en arándanos sin tratamiento térmico

identificó la flora fúngica presente en frutillas asintomáticas de la región centro-este de la provincia de Santa Fe. Nuestros resultados también concuerdan con Munitz y col. (2014) quién reportó que A. niger fue una de las principales especies fúngicas aisladas en arándanos cosechados en Concordia, Entre Ríos, entre 2009-2010, junto a otras aisladas en menor nivel como $A$. phaeospermum, Trichoderma harzianum, R. stolonifer, Botrytis cinerea, Alternaria vaccinii y A. flavus.

Nuestros resultados también fueron coincidentes con Olmedo y col. (2019) quienes aislaron e identificaron morfológicamente de frutos de arándanos frescos de la provincia de Tucumán los géneros fúngicos Fusarium sp. y Penicillium sp. En nuestro trabajo aislamos $F$. sporotrichioides, $P$. pinophilum, P. decumbens y P. purpurogenum. Este autor también reportó la presencia de Botrytis sp., Botryosphaeria sp., Cladosporium sp., Colletotrichum sp. y Pestalotia sp. que no fueron encontradas en nuestro trabajo.

\section{Especies de mohos aisladas en arándanos CTT}

En la Figura 2 se pueden observar los valores de Ab y Fr obtenidos para las especies de mohos aisladas de arándanos CTT.

Con los datos obtenidos tanto de $\mathrm{Fr}$ como de Ab podemos decir que la especie A. phaeospermun presenta el mayor porcentaje en ambos casos, siendo el más frecuente (30\%) y abundante (36\%) de todas las especies aisladas en las muestras de arándanos CTT analizadas. E. repens, sigue en segundo lugar con valores de $\mathrm{Fr}$ y $\mathrm{Ab}$ de $13 \%$ y $21 \%$, respectivamente. En tercer lugar se evidenció que $N$. fischeri alcanzó un valor de $\mathrm{Ab}$ de $12 \%$, pero la $\mathrm{Fr}$, al igual que para las demás especies aisladas fue menor al $10 \%$. 


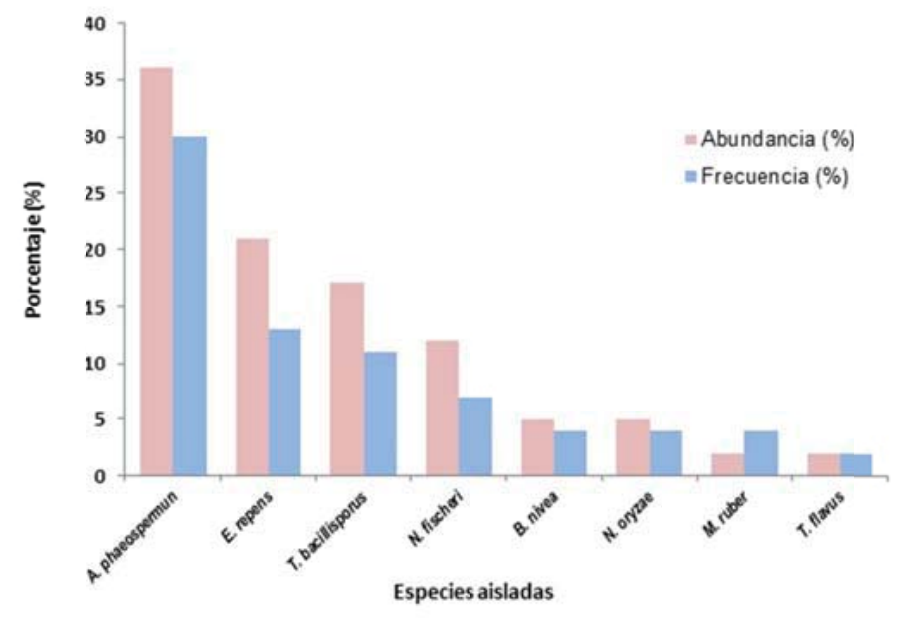

Figura 2. Abundancia y Frecuencia (\%) de mohos aislados en arándanos con tratamiento térmico

\section{DISCUSIÓN}

Los resultados obtenidos en relación a los mohos aislados de las muestras de arándanos frescos STT, evidenciaron que las especies más frecuentes y abundantes fueron A. niger (Fr: 70\% y Ab: 31\%) y $P$. decumbens (Fr: 21\% y Ab: 11\%). Mientras que para las muestras de arándanos CTT, las más frecuentes y abundantes fueron $A$. phaeospermun (Fr: 30\% y Ab: 36\%) у E. repens (Fr: 13\% y Ab: 21\%).

Wright y col. (2008) identificaron y determinaron la incidencia de mohos en frutos asintomáticos de arándano antes de su refrigeración, obteniendo como resultados presencia de Aspergillus, Penicillium y Rhízopus, similarmente a lo determinado en este trabajo para las muestras STT. Estos autores también determinaron la presencia de Alternaria, B. cinerea, Tríchoderma y Colletotríchum.
Nuestros resultados son coincidentes con Set Pérez y col. (2011) quienes aislaron hongos asociados a síntomas de pudrición del fruto de arándanos en Chile, siendo $B$. cinerea, A. alternata, C. herbarum y Stemphylium botryosum los de mayor incidencia; pero también detectaron, en menor proporción $R$. stolonifer, C. gloesporoides, Penicillium spp., Epicoccum nigrum y Fusarium sp. En el presente trabajo se aislaron con mayor Fr 3 especies del género Penicillium tales como $P$. decumbens, $P$. purpurogenum y $P$. pinophilum, y una especie de Fusarium (F. sporotrichioides).

Munitz (2013) en su estudio realizado en arándanos de la variedad Misty aisló diferentes especies como: A. phaeospermum, $C$. cladosporioides, Curvularia lunata, E. nigrum, E. chevalieri, Geotrichum candidum, Mucor racemosus, Trichocladium spp. y T. harzianum. El análisis de la Fr, mostró que C. gloeosporioides, A. tenuissima, Rhodotorula spp, A. niger, E. nigrum y Phomopsis 
vaccinii fueron los mohos predominantes sobre los arándanos de la variedad Misty contaminantes de dicha cosecha. Nuestro estudio se realizó sobre arándanos de la especie corymbosum, variedad $\mathrm{O}^{\prime} \mathrm{Neal}$, y coincidente con este autor también se aisló A. phaeospermum y una especie del género Eurotium, E. repens, diferente a la aislada por Munitz (2013), (E. chevalieri). Este autor también reportó la presencia de $A$. niger, especie también encontrada en nuestro trabajo con una Fr del 70\%. Por otra parte, Olmedo y col. (2019) aislaron e identificaron morfológicamente de frutos de arándanos naturalmente infectados de la provincia de Tucumán las siguientes especies fúngicas: Botrytis sp., Botryosphaeria sp., Cladosporium sp., Colletotrichum sp., Fusarium sp., Penicillium sp. y Pestalotia sp.

\section{CONCLUSIONES}

Se aislaron e identificaron ocho especies de mohos en arándanos frescos y ocho especies de mohos en arándanos tratados térmicamente (mohos termorresistentes). Los mohos aislados de arándanos frescos STT fueron: A. phaeospermun, A. niger, F. sporotrichioides, $P$. decumbens, $P$. pinophilum, $P$. purpurogenum, $R$. microsporus y $R$. oryzae.

Las especies aisladas de arándanos CTT fueron: A. phaeospermun, B. nívea, E. repens, M. ruber, $N$. fischeri, $N$. oryzae, T. bacillisporus y $T$. flavus.

Se determinó tanto la Fr como la Ab de los mohos aislados e identificados. Las especies de mohos aislados de arándanos frescos STT más frecuentes y abundantes fueron $A$. niger (Fr: 70\% y Ab: 31\%) y P. decumbens
(Fr: 21\% y Ab: 11\%). Las especies de mohos aislados de arándanos tratados térmicamente (termorresistentes) más frecuentes y abundantes fueron A. phaeospermun (Fr: 30\% y Ab: $36 \%$ ) y E. repens (Fr: $13 \%$ y Ab: $21 \%$ ).

Los resultados obtenidos en este estudio sirven como base para el diseño de tratamientos postcosecha que permitan reducir los mohos más frecuentes y abundantes presentes tanto en arándanos frescos como en aquellos destinados a procesamientos que incluyan una etapa de tratamiento térmico.

\section{AGRADECIMIENTOS}

Los autores agradecen el financiamiento de la Universidad Nacional del Litoral, Santa Fe, Argentina, a través del Proyecto CAI+D Código: 50120110100375 "Estrategias tecnológicas en el procesamiento de frutas frescas cortadas para incrementar su potencial saludable” y PICT 2017-406.

\section{BIBLIOGRAFÍA}

1.- AZEVEDO, J; FERNANDES, I.; FARIA, A; OLIVEIRA, J.; FERNANDES, A.; FREITAS, V.; MATEUS, N. 2010. Antioxidant properties of anthocyanidins, anthocyanidin-3-glucosides and respective portisins. Food Chemistry 119: 518-523.

2.- BEUCHAT, L.; COUSIN, M. 2002. Yeast and molds. En Compendium of methods for the microbiological examination of foods. Eds. Downes, F.; Keith, I. 4th Edition. American Public Health Association, Washington. D.C. p. 209 - 215.

3.- BOMBELLI, E.; MOSCHINI, R.; WRIGHT, E.; LOPEZ, M.; FABRIZIO, M. 2013. Modelado para la predicción de enfer- 
medades en cultivos de alto valor comercial. Proyecciones. 11 (1): 47-59.

4.- CANO, M.; SÁNCHEZ-MORENO, C; de PASCUAL-TERESA, S; de ANCOS, B. 2005. Capitulo7. Procesado Mínimo y Valor Nutricional. En: Nuevas Tecnologías de Conservación de Productos Vegetales Frescos Cortados. Ed. González-Aguilar, G.A.; Gardea, A.A.; Cuamea-Navarro, F.México. pp:155-176.

5.- DIVO DE SESAR, M.; PEPE, F.; MASSARA, D.; KAEN, R.; LOPEZ, V.; STELLA, A.; VILELLA, F. 2008. Variación en el contenido de polifenoles y antocianinas en frutos de los géneros Vaccinium y Rubus asociados a diversos factores condicionantes En: Divo de Sesar M.; Rocca M.; Vilella F. Avances en cultivos frutales no tradicionales. Arándanos - Cerezas - Frutillas - Granadas. Ed. Buenos Aires - Facultad de Agronomía, 153-165.

6.- FRISÓN, L.; CHIERICATTI, C. 2009.La sanitización en la industria alimentaria. RevistaCicha. Rosario, Santa Fe, Argentina. 78: 38-43.

7.- FRISÓN, L.; SOBRERO, S.; De JESÚS, J.; BASÍLICO, M.; BASÍLICO, J. 2012. Identificación y caracterización de especies de Neosartorya aisladas de frutillas (Fragaria spp.) frescas y tratadas térmicamente. Revista Venezolana de Ciencia y Tecnología de Alimentos. 4(1): 119-131.

8.- GARMENDIA, G.; VERO, S. 2006. Métodos para la desinfección de frutas y hortalizas. Horticultura. 197: 18-27.

9.- GIL, M.; ALLENDE, A.; MARTÍNEZ-SANCHEZ, A. 2007. Factores que afectan al contenido de compuestos bioactivos en alimentos de IV gama. V Congreso Iberoamericano de Tecnología Postcosecha y Agroexportaciones.
10.- GORDÓ, M. 2011. Guía práctica para el cultivo de Arándanos en la zona de la provincia de Buenos Aires. INTA San Pedro. Disponible en Web: https://inta.gob.ar/sites/ default/files/script-tmp-mg_0801.pdf. Consultada 2018.

11.- HINE, A.; ABDELNOUR, A. 2013. Establecimiento in vitro de arándano (Vacciniumcorymbosum L). Tecnología en Marcha, 26 (4): 64-71.

12.- JIN, P.; WANG, S. WANG, C.; ZHENG, Y. 2011. Effect of cultural system and storage temperature on antioxidant capacity and phenolic compounds in strawberries. In: Food Chemistry, 124: 262-270.

13.- KEVIN STEVE COSTAVALENTE BURGOS, K.; RUIZ REYES, S.; GANOZA YUPANQUI, M. 2016. Antocianinas totales y capacidad antioxidante in vitro de extractos de diferente grado etanólico del fruto de Vacinium corymbosum “Arandano”.UCV Scientia 8 (1): 44-48.

14.- LÓPEZ ALARCÓN, C.; DENICOLA, A. 2013. Evaluating the antioxidant capacity of natural products: A review on chemical and celular-based assay, in: AnalytucaChimicaActa, 763(6):1-10.

15.- MUNITZ, M. 2013. Arándanos: micoflora contaminante, micotoxinas, residuos de fungicidas y cinéticas de degradación. Tesis Doctoral en Química Industrial, Facultad de Ciencias Exactas y Naturales, Universidad de Buenos Aires, Buenos Aires, Argentina.

16.- MUNITZ, M.; MEDINA, M.; VISCIGLIO, S.; GIMENEZ, F.; RAVIOL, F.; SUBOVICH, G.; WILLIMAN, C.; GONZALEZ, A.; EVANGELINA MONTTI, $M$. 2014. Problemática en el muestreo postcosecha de arándanos para la determinación de fungicidas. Bistua Revista de la Facultad de Ciencias Básicas. 12(2): 48-57. ISSN 01204211. 
17.- OLMEDO G.M., HEREDIAA.M., NANNI M.L., VOLENTINI S.I. , RAPISARDA V.A., KIRSCHBAUM D. 2019. Actividad antifúngica de dióxido de cloro gaseoso contra hongos causantes de podredumbres poscosecha en arándano. Libro de resúmenes del II Congreso Argentino de Biología y Tecnología Poscosecha. Santiago del Estero, 11 al 13 de Septiembre de 2019. ISBN 978-9871676-88-0

18.- PIAGENTINI, A.M.; GÜEMES, D.R.; PIROVANI, M.E. 2003. Mesophilic aerobic population of fresh-cut spinach as affected by chemical treatment and type of packaging film. Journal of Food Science, 68(2), 602-607.

19.- PITT, J.; HOCKING, A. 2009. Fungi and Food Spoilage. $3^{\circ}$ Ed. Springer. New York. USA. 512 pp. ISBN 978-0-387-92206-5.

20.- PLANCICH, R. 2012. Identificación de la flora fúngica presente en frutillas naturales y estudio del efecto de la temperatura para prolongar su vida útil. Tesis de Licenciatura en ciencia y Tecnología de Alimentos. Facultad de ingeniería Química. Universidad Nacional del Litoral. Santa Fe. Argentina.

21.- PRETELL VASQUEZ, C.; MARQUEZ VILLACORTA, L.; SICHE, R. 2016. Efecto del ozono gaseoso sobre las características fisicoquímicas, microbiológicas y apariencia general de Punica Granatum L. wonderful fresca. Scientia Agropecuaria. 7: 173-180.

22.- RIVADENEIRA, M. 2010. Comportamiento productivo de frutales en Concordia. $3^{\circ}$ Jornada de Frutales Alternativos en Federal. INTA EEA Concordia, Entre Ríos, Argentina.

23.- RIVADENEIRA, M.; KIRSCHBAUM, D. 2011. INTA - Programa Nacional Frutales - Cadena arándano. Disponible en Web: http://inta.gob.ar/documentos/cadena-arandano-en-argentina/. Consultada: Febrero 2018.
24.- SAGPyA. 2016. Secretaria de Agricultura, Ganadería, Pesca y Alimentos, Argentina. Sector Frutas Finas. Arándano. Disponible en Web: http://www.minagri.gob.ar/SaGPyA/economias_regionales/_frutas/_informes/08_frutas_01_10.pdf. Consultada: 2016.

25.- SAMSON, R.; REENEN-HOEKSTRA, V.; HARTOG, B. 1992. Influence of pretreatment of raspberry pulp on the detection of heat resistant moulds. In Modern Methods in Food Mycology Eds.: Samson R., Hocking A., Pitt J. and King A. Elsevier, Amsterdam. p. 155 - 158.

26.- SET PÉREZ, F.; ENRIQUE FERRADA, Q.; JAIME GUERRERO, C.; EMMA BENSH. 2011. Berries\&Cherries, Revista frutícola del sur de Chile. http://revista.berriesandcherries.cl.

27.- UNDURRAGA, P., y VARGAS, S. (eds.). 2013. Manual del arándano. Boletín INIA $\mathrm{N}^{\circ}$ 263. Instituto de Investigaciones Agropecuarias INIA, Centro Regional de Investigación Quilamapu, Chillán, Chile. ISSN 0717 - 4829.

28.- VICENSINI, A.; ZAPATA DE BASÍLICO, M.; BASÍLICO, J. C. 1999. Estudio de contaminación fúngica de frutillas y productos derivados. FABICIB. 2: 91-97.

29.- WRIGHT, E.; FERNÁNDEZ, R.; BENYA, M.; RIVERA, M.; RONCORONI, S.; NICOLINI, F.; VÁSQUEZ, P.; CIURCA, P.; PÉREZ, B. 2008. Deterioro postcosecha de arándano en Entre Ríos y Buenos Aires, Argentina. Avances en cultivos frutales no tradicionales: arándanos, cerezas, kiwis, frutillas, granadas. P. 63-68. ISBN 978950-291065-9. 